\title{
Tubular Surface Having Pointwise 1-type Gauss Map in Euclidean 4-Space
}

\author{
İlim Kişi* and Günay Öztürk
}

(Communicated by Cihan Özgür)

\begin{abstract}
In this paper, we deal with a tubular surface in Euclidean 4-space $\mathbb{E}^{4}$. We study this surface with respect to its Gauss map. We show that there is not any tubular surface having harmonic Gauss map and we give the complete classification of tubular surface having pointwise 1-type Gauss map in Euclidean 4-space $\mathbb{E}^{4}$.
\end{abstract}

Keywords: tubular surface; Gauss map; pointwise 1-type.

AMS Subject Classification (2010): Primary: 53C40 ; Secondary: 53C42.

\section{Introduction}

The term of finite type immersions is presented by Chen, and then the same author writes some papers related to this topic [11, 12]. If a submanifold $M$ is given in Euclidean $m$-space $\mathbb{E}^{m}$, and if an isometric immersion $x: M \rightarrow \mathbb{E}^{m}$, also known as the position vector field of $M$, is written as a finite sum of eigenvectors of the Laplacian $\Delta$ of $M$ for a constant map $x_{0}$, and non-constant maps $x_{1}, x_{2}, \ldots, x_{k}$, i.e.,

$$
x=x_{0}+\sum_{i=1}^{k} x_{i},
$$

then $x$ is called as a finite type. Here, $\Delta x=\lambda_{i} x_{i}, \lambda_{i} \in \mathbb{R}, 1 \leq i \leq k$. If the numbers $\lambda_{i} \mathrm{~s}$ are different from each other, the submanifold is called as $k$-type [10].

This term is extended to the Gauss map of $M$ as

$$
\Delta G=a(G+C)
$$

for a real number $a$ and a constant vector $C$ by Chen and Piccinni in [13]. In this respect, a submanifold satisfying (1.1) is said to have 1-type Gauss map $G$. Then many papers have been written about submanifolds having 1-type Gauss map $G[4,5,6,23]$.

Afterwards, in (1.1), the real number $a$ is replaced with a non-constant function $f$. That is, the equation (1.1) becomes

$$
\Delta G=f(G+C)
$$

A submanifold satisfying (1.2) is said to have pointwise 1-type Gauss map $G$. If the function $f$ is non-constant, the pointwise 1-type Gauss map is called as proper. Also, if the vector $C$ is zero, the pointwise 1-type Gauss map is called as the first kind. Otherwise, second kind [14].

Surfaces satisfying (1.2) have been the subject of many studies such as [1, 15, 16, 17, 21, 22, 26, 27]. In recent years, authors deal with the meridian surfaces with pointwise 1-type Gauss map in some spaces in [2, 3].

When a space curve $\gamma(u)$, spine curve, is given, we can define a canal surface as the envelope of a one parameter family of spheres whose centers are the points of the spine curve $\gamma(u)$, and whose radii $r(u)$ are varying. If the radius function $r(u)$ is constant, the canal surface is called a tubular (tube) or a pipe surface. Actually, the notion of the canal surface is a generalization of an offset of a plane curve. In [18] and [19], some 
features of offset curves are discussed thoroughly. In [8], authors consider canal surfaces in $\mathbb{E}^{4}$. Also in [24, 25], the authors studied canal surfaces with parallel transport frame in $\mathbb{E}^{4}$.

In the present study, we consider tubular surface with respect to its Gauss map in $\mathbb{E}^{4}$. We find that there is no tubular surface having harmonic Gauss map and we give the complete classification of tubular surface with pointwise 1-type Gauss map in Euclidean 4 -space $\mathbb{E}^{4}$.

\section{Basic Concepts}

Given a regular surface $M$ in $\mathbb{E}^{4}$ with the parametrization $X(u, v):(u, v) \in D \subset \mathbb{E}^{2}$, at any point $p=X(u, v)$, the vectors $X_{u}$ and $X_{v}$ span the tangent space of $M$. Then the first fundamental form's coefficients are computed as

$$
E=\left\langle X_{u}, X_{u}\right\rangle, F=\left\langle X_{u}, X_{v}\right\rangle, G=\left\langle X_{v}, X_{v} .\right\rangle
$$

Here, $\langle$,$\rangle is the Euclidean inner product. For the regularity of the surface patch X(u, v), W^{2}=E G-F^{2} \neq 0$.

At any point $p$ in $M$, there is a decomposition $T_{p} \mathbb{E}^{4}=T_{p} M \oplus T_{p}^{\perp} M$, where $T_{p}^{\perp} M$ is the orthogonal component of $T_{p} M$ in $\mathbb{E}^{4}$. Let $\widetilde{\nabla}$ be the Riemannian connection of $\mathbb{E}^{4}$. Then the induced Riemannian connection on $M$ for any given local vector fields $X, Y$ tangent to $M$ is defined as

$$
\nabla_{X} Y=\left(\widetilde{\nabla}_{X} Y\right)^{T}
$$

where $T$ represents the tangential component.

Let $\chi(M)$ and $\chi^{\perp}(M)$ be the spaces of the smooth vector fields tangent and normal to $M$, respectively. The second fundamental map is defined as follows:

$$
h: \chi(M) \times \chi(M) \rightarrow \chi^{\perp}(M), h(X, Y)=\widetilde{\nabla}_{X} Y-\nabla_{X} Y .
$$

This map is well-defined, symmetric and bilinear. The equation (2.2) is known as the Gauss equation.

For each $X \in \chi(M)$ and $\xi \in \chi^{\perp}(M)$, the shape operator of $M$ is defined as

$$
\begin{aligned}
A & : \quad \chi^{\perp}(M) \times \chi(M) \rightarrow \chi(M) \\
A_{\xi} X & =-\left(\widetilde{\nabla}_{X} \xi\right)^{T}=-\widetilde{\nabla}_{X} \xi+\nabla_{X}^{\perp} \xi,
\end{aligned}
$$

where $A_{\xi}$ is the shape operator tensor, $\nabla^{\perp}$ is the normal connection belongs to $\chi^{\perp}(M)$. For any $X, Y \in \chi(M)$,

$$
\left\langle A_{\xi} X, Y\right\rangle=\langle h(X, Y), \xi\rangle
$$

holds. The operator $A_{\xi}$ is self-adjoint and bilinear. The equation (2.3) is known as the Weingarten equation [9]. Thus, the coefficients of the second fundamental forms of $M$ can be defined as follows:

$$
h_{i j}^{k}=\left\langle h\left(X_{i}, X_{j}\right), N_{k}\right\rangle, 1 \leq k \leq 2,
$$

where $X_{i}$ and $X_{j}$ are the orthonormal vectors of $T_{p} M$ [9].

The shape operator matrix corresponded to normal vector $N_{k}$ of $M$ is given

$$
A_{N_{k}}=\left(\begin{array}{cc}
h_{11}^{k} & h_{12}^{k} \\
h_{12}^{k} & h_{22}^{k}
\end{array}\right)
$$

[7]. The Gaussian curvature and the mean curvature vector of $M$ are given as

$$
K=\operatorname{det}\left(A_{N_{1}}\right)+\operatorname{det}\left(A_{N_{2}}\right),
$$

and

$$
\vec{H}=\frac{1}{2}\left\{i z\left(A_{N_{1}}\right) N_{1}+i z\left(A_{N_{2}}\right) N_{2}\right\},
$$

respectively [9].

Now, let us recall some basic concepts of the Gauss map of a submanifold $M$ in $\mathbb{E}^{m}$. The Grassmannian manifold, $G(n, m)$, consists of all oriented $n$-planes through the origin of $\mathbb{E}^{m}$ and the vector space $\wedge^{n} \mathbb{E}^{m}$, 
obtained by the exterior product of $n$-vectors in $\mathbb{E}^{m}$, can be defined as an Euclidean space $\mathbb{E}^{N}$, where $N=\left(\begin{array}{c}m \\ n\end{array}\right)$. In the light of this information, we can define the Gauss map. Let $\left\{e_{1}, e_{2}, \ldots, e_{n}, e_{n+1}, \ldots, e_{m}\right\}$ be an adapted orthonormal frame field in $\mathbb{E}^{m}$ such that $e_{1}, e_{2}, \ldots, e_{n}$ are tangent and $e_{n+1}, \ldots, e_{m}$ are normal to $M$, respectively. The map $G: M \rightarrow G(n, m) \subset \mathbb{E}^{N}$ which is defined as $G(p)=\left(e_{1} \wedge e_{2} \wedge \ldots \wedge e_{n}\right)(p)$ is called the Gauss map of $M$. This is a smooth map and assigns a point $p$ into an oriented $n$-plane in $\mathbb{E}^{m}$ by the parallel translation of the tangent space of $M$ at $p$ in $\mathbb{E}^{m}$.

For any real function $f$ on $M$, the Laplacian $\Delta f$ of $f$ is defined as

$$
\Delta f=-\sum_{i}\left(\widetilde{\nabla}_{e_{i}} \widetilde{\nabla}_{e_{i}} f-\widetilde{\nabla}_{\nabla_{e_{i}} e_{i}} f\right) .
$$

\section{Tubular Surfaces Having Pointwise 1-Type Gauss Map in $\mathbb{E}^{4}$}

Let $\gamma(u)=\left(f_{1}(u), f_{2}(u), f_{3}(u), 0\right)$ be a curve parametrized by arclength. The corresponding Frenet formulas have the following form:

$$
\begin{aligned}
\gamma^{\prime}(u) & =t(u), \\
t^{\prime}(u) & =\kappa(u) n(u), \\
n^{\prime}(u) & =-\kappa(u) t(u)+\tau(u) b_{1}(u), \\
b_{1}^{\prime}(u) & =-\tau(u) n(u), \\
b_{2}^{\prime}(u) & =0
\end{aligned}
$$

where $\left\{t(u), n(u), b_{1}(u), b_{2}(u)\right\}$ is the Frenet orthonormal basis of $\gamma$. The canal surface in $\mathbb{E}^{4}$ has the following parametrization (see [20]):

$$
M: X(u, v)=\gamma(u)+r(u)\left(b_{1}(u) \cos v+b_{2}(u) \sin v\right) .
$$

Now, we consider the surface $M$ in (3.2) as a tubular surface. Then its parametrization is given by

$$
M: X(u, v)=\gamma(u)+r\left(b_{1}(u) \cos v+b_{2}(u) \sin v\right),
$$

where $r$ is a real constant. For the vector fields $X_{1}, X_{2}$ are tangent, and $N_{1}, N_{2}$ are normal to $M$, we can choose an orthonormal frame $X_{1}, X_{2}, N_{1}, N_{2}$ given as in the following:

$$
\begin{aligned}
X_{1} & =\frac{X_{u}}{\left\|X_{u}\right\|}=\frac{t-(r \tau \cos v) n}{\sqrt{E}}, \\
X_{2} & =\frac{X_{v}}{\left\|X_{v}\right\|}=-(\sin v) b_{1}+(\cos v) b_{2}, \\
N_{1} & =(\cos v) b_{1}+(\sin v) b_{2}, \\
N_{2} & =\frac{(r \tau \cos v) t+n}{\sqrt{E}} .
\end{aligned}
$$

Here, $E=1+r^{2} \tau^{2} \cos ^{2} v$ is the coefficient of the first fundamental form. Moreover, by differentiating (3.4) covariantly with respect to $X_{1}$ and $X_{2}$, we obtain the following derivative formulas

$$
\begin{aligned}
\widetilde{\nabla}_{X_{1}} X_{1} & =a_{1} X_{2}+a_{2} N_{1}+a_{3} N_{2}, \\
\widetilde{\nabla}_{X_{1}} X_{2} & =-a_{1} X_{1}+a_{4} N_{2}, \\
\widetilde{\nabla}_{X_{1}} N_{1} & =-a_{2} X_{1}+a_{5} N_{2}, \\
\widetilde{\nabla}_{X_{1}} N_{2} & =-a_{3} X_{1}-a_{4} X_{2}-a_{5} N_{1}, \\
\widetilde{\nabla}_{X_{2}} X_{1} & =a_{4} N_{2}, \\
\widetilde{\nabla}_{X_{2}} X_{2} & =-\frac{1}{r} N_{1}, \\
\widetilde{\nabla}_{X_{2}} N_{1} & =\frac{1}{r} X_{2}, \\
\widetilde{\nabla}_{X_{2}} N_{2} & =-a_{4} X_{1},
\end{aligned}
$$


where

$$
\begin{aligned}
& a_{1}(u, v)=\frac{r \tau^{2} \cos v \sin v}{E}, \\
& a_{2}(u, v)=\frac{-r \tau^{2} \cos ^{2} v}{E}, \\
& a_{3}(u, v)=\frac{\kappa E-r \tau^{\prime} \cos v}{E^{\frac{3}{2}}}, \\
& a_{4}(u, v)=\frac{\tau \sin v}{E}, \\
& a_{5}(u, v)=\frac{-\tau \cos v}{E}
\end{aligned}
$$

are differentiable functions.

From the equation (2.4), the coefficients of the second fundamental form become

$$
h_{i j}^{k}=\left\langle h\left(X_{i}, X_{j}\right), N_{k}\right\rangle=\left\langle A_{N_{k}} X_{i}, X_{j}\right\rangle, 1 \leq i, j \leq 2, k=3,4 .
$$

By considering the equations (2.2), (3.4), and (3.7), we obtain the coefficients

$$
\begin{aligned}
& h_{11}^{1}=a_{2}, h_{12}^{1}=0, h_{22}^{1}=-\frac{1}{r}, \\
& h_{11}^{2}=a_{3}, h_{12}^{2}=a_{4}, h_{22}^{2}=0 .
\end{aligned}
$$

Lemma 3.1. Let $M$ be a tubular surface given with the parametrization (3.3) in $\mathbb{E}^{4}$. Then the shape operator matrices are given as follows:

$$
A_{N_{1}}=\left[\begin{array}{cc}
a_{2} & 0 \\
0 & -\frac{1}{r}
\end{array}\right], \quad A_{N_{2}}=\left[\begin{array}{cc}
a_{3} & a_{4} \\
a_{4} & 0
\end{array}\right] .
$$

In the following corollary, we obtain the special cases of the results which have been given in [8] with a different calculation method.

Corollary 3.1. [8] Let $M$ be a tubular surface given with the parametrization (3.3) in $\mathbb{E}^{4}$. The Gaussian curvature, and the mean curvature vector of $M$ are respectively given by

$$
K=\frac{2 \tau^{2} \cos ^{2} v+r^{2} \tau^{4} \cos ^{4} v-\tau^{2}}{E^{2}}
$$

and

$$
\vec{H}=\frac{1}{2}\left\{\left(\frac{-2 r^{2} \tau^{2} \cos ^{2} v-1}{E r}\right) N_{1}+\left(\frac{\kappa E-r \tau^{\prime} \cos v}{E^{\frac{3}{2}}}\right) N_{2}\right\} .
$$

Proof. From the Lemma 3.1, using the equations (2.7), and (2.8), we get

$$
\begin{aligned}
K & =\operatorname{det}\left(A_{N_{1}}\right)+\operatorname{det}\left(A_{N_{2}}\right) \\
& =\frac{\tau^{2} \cos ^{2} v}{E}-\frac{\tau^{2} \sin ^{2} v}{E^{2}} \\
& =\frac{2 \tau^{2} \cos ^{2} v+r^{2} \tau^{4} \cos ^{4} v-\tau^{2}}{E^{2}},
\end{aligned}
$$

and

$$
\begin{aligned}
\vec{H} & =\frac{1}{2}\left\{i z\left(A_{N_{1}}\right) N_{1}+i z\left(A_{N_{2}}\right) N_{2}\right\} \\
& =\frac{1}{2}\left\{\left(\frac{-r \tau^{2} \cos ^{2} v}{E}-\frac{1}{r}\right) N_{1}+\left(\frac{\kappa E-r \tau^{\prime} \cos v}{E^{\frac{3}{2}}}\right) N_{2}\right\} \\
& =\frac{1}{2}\left\{\left(\frac{-2 r^{2} \tau^{2} \cos ^{2} v-1}{E r}\right) N_{1}+\left(\frac{\kappa E-r \tau^{\prime} \cos v}{E^{\frac{3}{2}}}\right) N_{2}\right\} .
\end{aligned}
$$


The Gauss map of a given surface $M$ in $\mathbb{E}^{4}$ is defined by $G=X_{1} \wedge X_{2}$. Using the equations (3.5), and a straight-forward computation, we get the Laplacian of the Gauss map as follows:

$$
\begin{aligned}
\Delta G= & \left(a_{2}^{2}+a_{3}^{2}+2 a_{4}^{2}+\frac{1}{r^{2}}\right) X_{1} \wedge X_{2} \\
& +\left(-a_{1} a_{2}+a_{4} a_{5}-\frac{a_{1}}{r}\right) X_{1} \wedge N_{1} \\
& +\left(-X_{1}\left[a_{4}\right]-a_{1} a_{3}\right) X_{1} \wedge N_{2} \\
& +\left(X_{1}\left[a_{2}\right]-a_{3} a_{5}\right) X_{2} \wedge N_{1} \\
& +\left(X_{1}\left[a_{3}\right]+a_{2} a_{5}-2 a_{1} a_{4}+X_{2}\left[a_{4}\right]\right) X_{2} \wedge N_{2} \\
& +\left(-2 a_{2} a_{4}-\frac{2 a_{4}}{r}\right) N_{1} \wedge N_{2},
\end{aligned}
$$

where $X_{i}\left[a_{j}\right](i=1,2 ; j=2,3,4)$ is the directional derivative with respect to $X_{i}$.

Corollary 3.2. There is no tubular surface having harmonic Gauss map.

Proof. Assume that the surface $M$ has harmonic Gauss map, i.e., $\Delta G=0$. From (3.9), we have $a_{2}^{2}+a_{3}^{2}+2 a_{4}^{2}+$ $\frac{1}{r^{2}}=0$, a contradiction. Hence, there is no tubular surface having harmonic Gauss map.

Now, we assume that the tubular surface $M$ given by the parametrization (3.3) has pointwise 1-type Gauss map. From (1.2), and (3.9), the equations

$$
\begin{aligned}
f+f\left\langle C, X_{1} \wedge X_{2}\right\rangle & =a_{2}^{2}+a_{3}^{2}+2 a_{4}^{2}+\frac{1}{r^{2}}, \\
f\left\langle C, X_{1} \wedge N_{1}\right\rangle & =-a_{1} a_{2}+a_{4} a_{5}-\frac{a_{1}}{r}, \\
f\left\langle C, X_{1} \wedge N_{2}\right\rangle & =-X_{1}\left[a_{4}\right]-a_{1} a_{3}, \\
f\left\langle C, X_{2} \wedge N_{1}\right\rangle & =X_{1}\left[a_{2}\right]-a_{3} a_{5}, \\
f\left\langle C, X_{2} \wedge N_{2}\right\rangle & =X_{1}\left[a_{3}\right]+a_{2} a_{5}-2 a_{1} a_{4}+X_{2}\left[a_{4}\right], \\
f\left\langle C, N_{1} \wedge N_{2}\right\rangle & =-2 a_{2} a_{4}-\frac{2 a_{4}}{r}
\end{aligned}
$$

hold. Here, $f$ is a non-zero smooth function. Using the equation (1.2), we can write

$$
\begin{aligned}
C & =A_{1} X_{1} \wedge X_{2}+A_{2} X_{1} \wedge N_{1}+A_{3} X_{1} \wedge N_{2} \\
& +A_{4} X_{2} \wedge N_{1}+A_{5} X_{2} \wedge N_{2}+A_{6} N_{1} \wedge N_{2}
\end{aligned}
$$

where

$$
\begin{aligned}
& A_{1}(u, v)=\frac{a_{2}^{2}+a_{3}^{2}+2 a_{4}^{2}+\frac{1}{r^{2}}}{f}-1, \\
& A_{2}(u, v)=\frac{-a_{1} a_{2}+a_{4} a_{5}}{f}-\frac{a_{1}}{f r}, \\
& A_{3}(u, v)=\frac{-X_{1}\left[a_{4}\right]-a_{1} a_{3}}{f}, \\
& A_{4}(u, v)=\frac{X_{1}\left[a_{2}\right]-a_{3} a_{5}}{f}, \\
& A_{5}(u, v)=\frac{X_{1}\left[a_{3}\right]+a_{2} a_{5}-2 a_{1} a_{4}+X_{2}\left[a_{4}\right]}{f}, \\
& A_{6}(u, v)=\frac{-2 a_{2} a_{4}}{f}-\frac{2 a_{4}}{f r}
\end{aligned}
$$

are differentiable functions.

In the following theorems, we give some results about tubular surfaces having pointwise 1-type Gauss map of the first and the second kinds. 
Theorem 3.1. Let $M$ be a tubular surface given with the parametrization (3.3) in $\mathbb{E}^{4}$. If $M$ has pointwise 1-type Gauss map of the first kind, then $M$ is one of the following surfaces in $\mathbb{E}^{4}$ :

(i) circular cylinder,

(ii) torus with the parametrization,

$$
M: X(u, v)=(\cos u, \sin u, r \cos v, r \sin v) .
$$

Proof. Let $M$ be a tubular surface given with the parametrization (3.3) in $\mathbb{E}^{4}$. We suppose that $M$ has pointwise 1-type Gauss map of the first kind, i.e. $C$ is zero. From the equations (3.10), and (3.11), we have $-2 a_{2} a_{4} r-2 a_{4}=$ 0 , so $a_{4}=0$, which implies $\tau=0$. Writing $\tau=0$ in the equations (3.6), we obtain $a_{1}=a_{2}=a_{5}=0$, and $a_{3}=\kappa$. Since the coefficient of $X_{2} \wedge N_{2}$ is zero, $X_{1}\left[a_{3}\right]=\kappa^{\prime}=0$.

Then there are two cases: $\kappa=0$ or $\kappa$ is constant. If $\kappa=0, \gamma$ is a straight line and $M$ is circular cylinder. If $\kappa$ is constant, $\gamma$ is a circle and $M$ is a torus with the parametrization (3.12).

Corollary 3.3. If $M$ has pointwise 1-type Gauss map of the first kind, then the function $f$ is constant, and expressed as $f=\kappa^{2}+\frac{1}{r^{2}}$. In this case, the Gauss map is not proper.

Proof. Let $M$ has pointwise 1-type Gauss map of the first kind. Since $A_{1}$ is zero, from the equations (3.11),

$$
\frac{a_{2}^{2}+a_{3}^{2}+2 a_{4}^{2}+\frac{1}{r^{2}}}{f}-1=0,
$$

i.e., $\kappa^{2}+\frac{1}{r^{2}}=f$. Since $\kappa$ and $r$ is constant, $f$ is constant and so the Gauss map is not proper.

Theorem 3.2. Let $M$ be a tubular surface given with the parametrization (3.3) in $\mathbb{E}^{4}$. If $M$ has pointwise 1-type Gauss map of the second kind,

$$
\begin{aligned}
X_{1}\left[A_{1}\right]-a_{4} A_{3}+a_{2} A_{4}+a_{3} A_{5} & =0, \\
X_{1}\left[A_{2}\right]-a_{5} A_{3}-a_{1} A_{4}+a_{3} A_{6} & =0, \\
X_{1}\left[A_{3}\right]+a_{4} A_{1}+a_{5} A_{2}-a_{1} A_{5}-a_{2} A_{6} & =0, \\
X_{1}\left[A_{4}\right]-a_{2} A_{1}+a_{1} A_{2}-a_{5} A_{5}+a_{4} A_{6} & =0, \\
X_{1}\left[A_{5}\right]-a_{3} A_{1}+a_{1} A_{3}+a_{5} A_{4} & =0, \\
X_{1}\left[A_{6}\right]-a_{3} A_{2}+a_{2} A_{3}-a_{4} A_{4} & =0,
\end{aligned}
$$

and

$$
\begin{aligned}
X_{2}\left[A_{1}\right]+a_{4} A_{5}+\frac{A_{2}}{r} & =0 \\
X_{2}\left[A_{2}\right]+a_{4} A_{6}-\frac{A_{1}}{r} & =0 \\
X_{2}\left[A_{3}\right] & =0, \\
X_{2}\left[A_{4}\right] & =0, \\
X_{2}\left[A_{5}\right]-a_{4} A_{1}+\frac{A_{6}}{r} & =0 \\
X_{2}\left[A_{6}\right]-a_{4} A_{2}-\frac{A_{5}}{r} & =0
\end{aligned}
$$

hold, where $X_{i}\left[A_{j}\right](i=1,2 ; j=1, \ldots, 6)$ is the directional derivative with respect to $X_{i}$.

Proof. Let $M$ has pointwise 1-type Gauss map of the second kind, i.e., $C$ is constant. Using (3.5), and (3.10), we get

$$
\begin{aligned}
\widetilde{\nabla}_{X_{1}} C= & \left(X_{1}\left[A_{1}\right]-a_{4} A_{3}+a_{2} A_{4}+a_{3} A_{5}\right) X_{1} \wedge X_{2} \\
& +\left(X_{1}\left[A_{2}\right]-a_{5} A_{3}-a_{1} A_{4}+a_{3} A_{6}\right) X_{1} \wedge N_{1} \\
& +\left(X_{1}\left[A_{3}\right]+a_{4} A_{1}+a_{5} A_{2}-a_{1} A_{5}-a_{2} A_{6}\right) X_{1} \wedge N_{2} \\
& +\left(X_{1}\left[A_{4}\right]-a_{2} A_{1}+a_{1} A_{2}-a_{5} A_{5}+a_{4} A_{6}\right) X_{2} \wedge N_{1} \\
& +\left(X_{1}\left[A_{5}\right]-a_{3} A_{1}+a_{1} A_{3}+a_{5} A_{4}\right) X_{2} \wedge N_{2} \\
& +\left(X_{1}\left[A_{6}\right]-a_{3} A_{2}+a_{2} A_{3}-a_{4} A_{4}\right) N_{1} \wedge N_{2}
\end{aligned}
$$


and

$$
\begin{aligned}
\widetilde{\nabla}_{X_{2}} C= & \left(X_{2}\left[A_{1}\right]+a_{4} A_{5}+\frac{A_{2}}{r}\right) X_{1} \wedge X_{2} \\
& +\left(X_{2}\left[A_{2}\right]+a_{4} A_{6}-\frac{A_{1}}{r}\right) X_{1} \wedge N_{1} \\
& +X_{2}\left[A_{3}\right] X_{1} \wedge N_{2} \\
& +X_{2}\left[A_{4}\right] X_{2} \wedge N_{1} \\
& +\left(X_{2}\left[A_{5}\right]-a_{4} A_{1}+\frac{A_{6}}{r}\right) X_{2} \wedge N_{2} \\
& +\left(X_{2}\left[A_{6}\right]-a_{4} A_{2}-\frac{A_{5}}{r}\right) N_{1} \wedge N_{2}
\end{aligned}
$$

Since $C$ is constant, then the coefficient of the vectors $\widetilde{\nabla}_{X_{1}} C$ and $\widetilde{\nabla}_{X_{2}} C$ must vanish. Thus, we have the equations (3.13) and (3.14) which completes the proof.

\section{References}

[1] Arslan, K., Bayram, B. K., Bulca, B., Kim, Y. H., Murathan, C. and Öztürk, G., Rotational embeddings in $\mathbb{E}^{4}$ with pointwise 1-type Gauss map. Turk. J. Math. 35 (2011), 493-499.

[2] Arslan, K., Bulca, B. and Milousheva, V., Meridian surfaces in $\mathbb{E}^{4}$ with pointwise 1-type Gauss map. Bull. Korean Math. Soc. 51 (2014), 911-922.

[3] Arslan, K. and Milousheva, V., Meridian surfaces of elliptic or hyperbolic type with pointwise 1-type Gauss map in Minkowski 4-space. Taiwanese J. Math. 20 (2016), 311-332.

[4] Baikoussis, C. and Blair, D. E., On the Gauss map of ruled surfaces. Glasgow Math. J. 34 (1992), 355-359.

[5] Baikoussis, C., Chen, B. Y. and Verstraelen, L., Ruled surfaces and tubes with finite type Gauss map. Tokyo J. Math. 16 (1993), 341-349.

[6] Baikoussis, C. and Verstraelen, L., On the Gauss map of helicoidal surfaces. Rend. Sem. Mat. Messina Ser. II 16 (1993), 31-42.

[7] Bulca, B., A characterization of surfaces in $\mathbb{E}^{4}$. PhD Thessis. 2012.

[8] Bulca, B., Arslan, K., Bayram, B. and Öztürk, G., Canal surfaces in 4-dimensional Euclidean space. IJOCTA 7 (2017), 83-89.

[9] Chen, B. Y., Geometry of Submanifolds. Dekker, New York, 1973.

[10] Chen, B. Y., Total Mean Curvature and Submanifolds of Finite Type. Series in Pure Mathematics. 1. World Scientific Publishing Co. Singapore, 1984.

[11] Chen, B. Y., A report on submanifolds of finite type. Soochow J. Math. 22 (1996), 117-337.

[12] Chen, B. Y., On submanifolds of finite type. Soochow J. Math. 9 (1983), 65-81.

[13] Chen, B. Y. and Piccinni, P., Submanifolds with finite type Gauss map. Bull. Austral. Math. Soc. 35 (1987), 161-186.

[14] Chen, B. Y., Choi, M. and Kim, Y. H., Surfaces of revolution with pointwise 1-type Gauss map. J. Korean Math. 42 (2005), $447-455$.

[15] Choi, M. and Kim, Y. H., Characterization of the helicoid as ruled surfaces with pointwise 1-type Gauss map. Bull. Korean Math. Soc. 38 (2001), 753-761.

[16] Dursun, U., On spacelike rotational surfaces with pointwise 1-type Gauss map. Bull. Korean Math. Soc. 52 (2015), 301-312.

[17] Dursun, U. and Arsan, G. G., Surfaces in the Euclidean space $\mathbb{E}^{4}$ with pointwise 1-type Gauss map. Hacettepe Journal of Mathematics and Statistics 40 (2011), 617-625.

[18] Farouki, R. T. and Neff, C. A., Analytic properties of plane offset curves. Computer-Aided Geometric Design 7 (1990), 83-99.

[19] Farouki, R. T. and Neff, C. A., Algebraic properties of plane offset curves. Computer-Aided Geometric Design 7 (1990), 101-127.

[20] Gal, R. O. and Pal, L., Some notes on drawing twofolds in 4-dimensional Euclidean space. Acta Univ. Sapientiae, Informatica 1 (2009), 125-134.

[21] Kim, Y. H. and Yoon, D. W., Ruled surfaces with pointwise 1-type Gauss map. J. Geom. Phys. 34 (2000), 191-205.

[22] Kim, Y. H. and Yoon, D. W., Classification of rotation surfaces in pseudo-Euclidean space. J. Korean Math. 41 (2004), 379-396.

[23] Kim, Y. H. and Yoon, D. W., Ruled surfaces with finite type Gauss map in Minkowski spaces. Soochow J. Math. 26 (2000), 85-96.

[24] Kişi, İ., Öztürk, G. and Arslan, K., A new type of canal surface in Euclidean 4-space $\mathbb{E}^{4}$. Sakarya University Journal of Science 23(5) (2019), 801-809.

[25] Kişi, İ. and Öztürk, G., A new approach to canal surface with parallel transport frame. Int. J. Geom. Methods Mod. Phys. 14 (2017), 17500261-1750026-16.

[26] Niang, A., Rotation surfaces with 1-type Gauss map. Bull. Korean Math. Soc. 42 (2005), 23-27.

[27] Yoon, D. W., On the Gauss map of translation surfaces in Minkowski 3-spaces. Taiwanese J. Math. 6 (2002), 389-398. 


\section{Affiliations}

İLIM KIŞI

ADDRESS: Kocaeli University, Dept. of Mathematics, 41380, Kocaeli-Turkey.

E-MAIL: ilim.ayvaz@kocaeli.edu.tr

ORCID ID: : 0000-0002-4785-8165

\section{GÜNAY ÖZTÜRK}

ADDRESS: İzmir Demokrasi University, Dept. of Mathematics, 35140, İzmir-Turkey.

E-MAIL: gunay.ozturk@idu.edu.tr

ORCID ID: : 0000-0002-1608-0354 